\title{
PENETRATING CORNEAL GRAFT* \\ ORIENTATION OF THE INITIAL PENETRATION OF THE TREPHINE INTO THE HOST CORNEA
}

BY

\author{
I. C. MICHAELSON \\ Haifa, Israel
}

DURING the operation of perforating graft of the cornea, the trephine in the great majority of cases enters the anterior chamber in only a portion of its circumference, the remaining circumference of the graft being completed with scissors. The author has noticed, both in operations performed personally and in those performed by others, that the portion of the circumference of the trephine which first enters the anterior chamber is usually that nearest to the wrist of the operating hand, despite the customary effort of making an even trephine cut. Since this was first noted, particular attention has been directed to it, and it has been found that in all of six perforating graft operations performed during a period of 3 weeks the trephine entered the anterior chamber on the wrist side.

To confirm this observation, sixteen enucleated eyes were trephined with the same care as would be used in the operating theatre for the recipient eye. That is, the trephine cut was stopped as soon as it was clear that the anterior chamber had been entered. In five cases the anterior chamber was entered practically simultaneously in the entire circumference of the trephine cut, and in the remaining eleven cases, the anterior chamber was entered nine times on the wrist side, and twice on the other side. The fairly large proportion of cases (six out of sixteen) in which the whole circumference of the trephine reached the anterior chamber simultaneously was probably due to the relative confidence of the laboratory as compared with the operating theatre.

It would appear that, so far as the author is concerned, there is a tendency to tilt the trephine in the direction of the wrist of the hand holding it, despite the effort to keep the instrument vertical.

\section{Application}

It was found that clinically, advantage might be taken of this circumstance in the following conditions:

(1) In cases in which a perforating graft operation is performed despite the presence of a small anterior synechia, the intention being to separate the synechia during the operation.

* Received for publication April 29, 1953. 
The synechia may have remained after a preliminary synechiotomy operation, and not be large enough to demand a further preliminary synechiotomy. In these cases it is easier to separate the synechia if the initial entry of the trephine into the anterior chamber is made in the sector of the cornea in which the

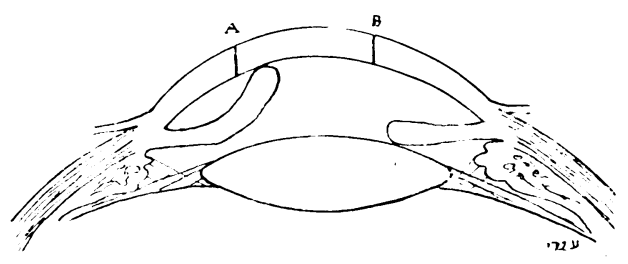

FIG. 1.-A and $B$ represent the limits of the portion of the host cornea which is to be removed. A is situated over the portion of the iris where there is a small anterior synechia. It is advisable that, if all the graft is not cut with the trephine simultaneously, the anterior chamber be entered at $A$ rather than at $B$. This permits easier and safer separation of the anterior synechia. The trephine is therefore held by the author so that his wrist is directed towards A.

synechia is situated. The reasons for this are as follows:

(a) The synechia should be separated before the scissor portion of the circumference is cut, thereby facilitating the latter manoeuvre. This can be done if the synechia is in the portion of the cornea where the initial entrance into the anterior chamber is made.

(b) It is better to approach a synechia from the limbal side rather than from the pupil side. The former procedure permits stretching the synechia, and at the same time the dividing instrument, scissor or spatula, is protected from the lens by the body of the iris (Fig. 1).

Therefore, the trephine should be held so that the surgeon's wrist is at that side of the trephine which is in the same meridian as the anterior synechia.

(2) In cases in which the trephine cut traverses opaque and transparent portions of the cornea.

It is advisable to have the trephine portion of the cut in the opaque area and the scissor portion in the transparent area. The reasons are these:

(a) The trephine portion of the cut heals quicker than the scissor portion and should therefore be sited in the opaque portion of the cornea which heals less quickly than the transparent portion.

(b) The scissor cut can be made more accurately in transparent cornea than in opaque cornea.

Therefore, if the cut must traverse opaque and transparent portions of a recipient cornea, the trephine should be held so that the surgeon's wrist is at that side of the trephine which lies over the opaque portion of the cornea (Fig. 2).

These small practical points are put forward because success or failure in graft operations rests in most cases upon scrupulous attention to detail. It is appreciated that the orientation of the initial penetration into the

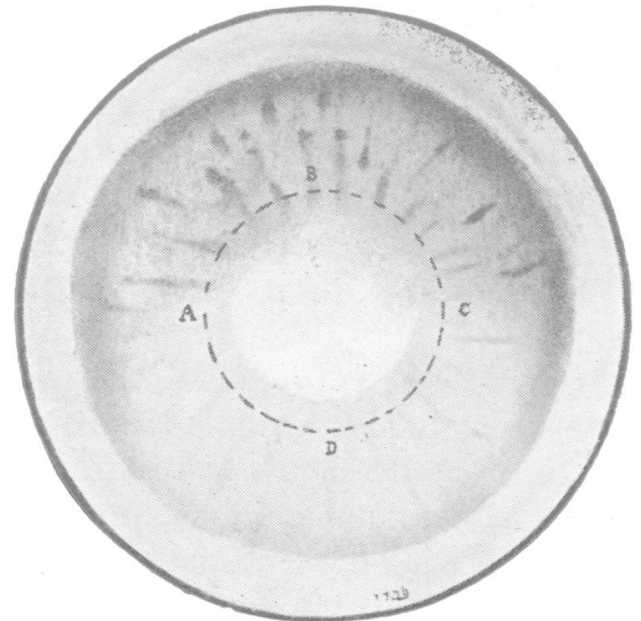

FIG. 2.-ABCD is the circumference of the portion of the host cornea which is to be removed. The portion ABC traverses relatively clear cornea, the portion ADC lies in dense opacity. It is advisable that if any portion must be cut with scissors it should be that corresponding to $\mathrm{ABC}$. The trephine is, therefore, held by the author so that his wrist is directed towards 6 o'clock. 
cornea according to the position of the surgeon's wrist may be peculiar to only a few. It is, however, suggested that each surgeon should examine his own proclivity in this direction, so that the tendency, if found, may be exploited clinically as described above.

\section{Summary}

(1) The meridian of the initial penetration of the trephine into the cornea is of some importance where an anterior synechia is present or where the circumference of the cut traverses both the clear and the opaque regions of the cornea.

(2) It is shown in the author's own case that the position of the wrist of the hand holding the trephine determines the meridian of the initial penetration of the trephine into the recipient cornea.

(3) It is suggested that others may find a personal tendency for the trephine to enter the anterior chamber in a meridian determined by the position of the hand holding the instrument.

This work has been supported by a grant from the National Council to combat Blindness, New York. 